\title{
Capture d'innovation : étude de cas d'une néo-banque à mission sociale et défis pour les pays en développement
}

\section{Recapturing Innovation: A case study of Neobanking with a social mission and the challenges of its business model for developing countries}

\author{
Arvind Ashta ${ }^{1}$, Djamchid Assadi $^{2}$, Nathalie Duran ${ }^{3}$ \\ ${ }^{1}$ CEREN EA 7477, Burgundy School of Business, Université Bourgogne Franche-Comté, Dijon, France, \\ Arvind.ashta@bsb-education.com \\ ${ }^{2}$ CEREN EA 7477, Burgundy School of Business, Université Bourgogne Franche-Comté, Dijon, France, \\ djamchid.assadi@bsb-education.com \\ ${ }^{3}$ CEMOI, IAE Réunion, Université de la Réunion, Saint Denis, France
}

RÉSUMÉ. Le concept de "capture d'innovation " explique pourquoi les opérateurs historiques permettent aux challengers d'utiliser des innovations radicales qui potentiellement perturbent le marché. Les challengers sont des petits acteurs agiles qui cherchent à prouver que leur innovation est viable, en passant par la vente à un acteur historique. Ce dernier peut ensuite récupérer les marchés perdus et utiliser ses compétences pour intensifier l'innovation capturer, préservant ainsi son avantage concurrentiel. Ce concept est illustré à travers l'étude de cas d'une néo-banque, innovation dans le paysage financier, car son principe est d'offrir une gamme limitée de produits uniquement via le téléphone portable. Le cas montre également que les challengers ont utilisé une nouvelle technologie, une forme organisationnelle différente et une innovation dans les canaux de distribution pour cibler des clients aux besoins non satisfaits. En Inde à cause des règlementations en vigueur un cas similaire n'a pas eu le succès escompté. C'est pourquoi il nous semble important que les organes de régulation dans les pays en développement veillent à avoir une réglementation appropriée pour faciliter la réussite des entreprises innovantes, et ne devraient pas bloquer leurs stratégies de prix, de promotion ni de distribution.. II est à composer en Arial 9, interligné 13 points.

ABSTRACT. The concept of "recapturing innovations" explains why incumbents allow challengers to use radical innovations to potentially disrupt the market. Challengers are small and nimble operators who seek high powered incentives to show that their innovations are viable, and the realizations of these objectives come from selling to an incumbent. Incumbents can then recapture their lost markets and use their efficient operating systems and regulatory knowledge to scale up the recaptured innovations, thus sustaining their competitive advantage. This concept is illustrated through a case study on a neobank, which is an innovation in the financial landscape, usually offering a limited range of products through mobile telephones. This case study shows that the challengers used a new organizational form, new technology and distribution channels in order to satisfy a large niche of unserved or underserved clients. This case is similar to the payment banks in India which have not fared well, perhaps because of too much regulatory interference. Therefore, regulatory agencies in developing countries should have appropriate regulations in order to reduce the institutional barriers hindering the success of social enterprises. However, they should not block initiatives in their product pricing, promoting and distributing policies.

MOTS-CLÉS. innovation disruptive, innovation radicale, innovation de maintien, innovation sociale, banque, Fintech, inclusion financière.

KEYWORDS. disruptive innovation, radical innovation, sustaining innovation, social innovation, banking, Fintech, financial inclusion.

\section{Introduction}

Le nombre de banques diminue massivement dans le monde. Certaines agences ferment, principalement dans les zones rurales et les petites villes pour réduire les coûts de distribution (cf annexe 1). De nouveaux modèles sans agence, à la fois numérique et à distance apparaissent peu à peu pour les remplacer. Un bon nombre de startups, surtout des Fintech, se créent des niches et grignotent des parts de marché aux banques traditionnelles en raison de l'utilisation intensive de la technologie 
mais aussi de plus faibles coûts [ASS 18] [CRO 15A]. Ces innovations disruptives ciblent également les petits marchés et ceux de bas de gamme qui sont souvent abandonnés par les banques traditionnelles intéressées principalement par des clients à plus forte valeur ajoutée [CHR 15].

Si les banques n'innovent pas pour être compétitives sur ces marchés, alors les acteurs Fintech qui sont leurs challengers pourraient les dépasser. Les banques peuvent bien évidemment racheter une Fintech afin de récupérer ses parts de marché. C'est cette stratégie que nous qualifions « capture d'innovation », analysée dans notre article à travers le cas de la banque de paiement Nickel, leader en France. Cette étude pourrait servir de modèle pour les pays en développement afin aider les entreprises du sociale à dépasser les barrières institutionnelles.

Le cas nous permet également de réfléchir à deux questions. Premièrement, alors que les opérations Fintech les plus réussies en Europe semblent venir d'Angleterre ou d'Allemagne, comment une Fintech française peut-elle garder une longueur d'avance sur ses concurrents britanniques et allemands ? Comment une innovation radicale peut-elle réussir à transformer la banque en France où les réglementations et les normes sont si rigides notamment dans le secteur bancaire? Si ce type d'innovation fonctionne en France, pourquoi cela ne pourrait-il pas en être de même dans les pays en développement?

Deuxièmement, comment une néo-banque française peut-elle offrir un service de paiement aux exclus financiers faute de surendettement, et aux pauvres, alors que les banques de paiement indiennes sont en grande difficulté malgré l'intérêt et le soutien des décideurs politiques [ASS 20B] ? Le cas Nickel représente un cas d'innovation sociale responsable [TEM 18] et commande l'objet et le déploiement de notre recherche.

Notre question de recherche est ainsi la suivante : comment une telle innovation française peut-elle être un succès malgré l'opposition conservatrice ? L'étude de cas nous permettra d'enrichir le cadre théorique de l'innovation et fournir des préconisations pour ce type d'innovations sociales dans les pays en voie de développement.

Ce papier s'organise comme suit : premièrement, nous présentons un aperçu de la littérature sur l'innovation en général et l'innovation sociale en particulier. Dans un deuxième temps, nous expliquons la méthodologie de recherche adoptée. Ensuite, nous étudions le cas de la néo-banque Nickel, sa stratégie et son modèle économique. Finalement, nous explorons les implications de ce cas pour la théorie de l'innovation et nous proposons des recommandations pour les pays en développement.

\section{Innovation, innovation sociale et la place de la néo-banque}

L'innovation est un sujet important pour la satisfaction des besoins sociaux. Prenons le cas de l'emploi qui implique l'inclusion sociale. Les entreprises qui innovent contribuent davantage à l'emploi que les entreprises non innovantes. Dans le pays médian du groupe OCDE, 53\% des entreprises avec au moins une innovation au cours de la période 2014-16 représentent 70\% de l'emploi, respectivement $56 \%$ et $77 \%$ pour la France [OEC 20]. Ces entreprises n'entreprennent forcément pas leur propre recherche et développement et peuvent bénéficier de la diffusion des innovations développées par d'autres. Dans les pays médians de l'OCDE, 25\% des entreprises innovantes bénéficient d'un soutien de l'État, on va jusqu'à $47 \%$ pour la France [OEC 20]. Le cas Nickel que nous étudions ici, révèle comment une innovation peut réussir en France sans aide de l'État et malgré l'opposition des conservateurs.

Notre question de recherche nous conduit à l'examen de la littérature de l'innovation, qui inclut plusieurs concepts [USU 18], en vue de réunir les éléments adéquats pour étudier une innovation techno-organisationnelle dans le secteur financier : la néo-banque. 
Dans cette section, nous étudions la théorie d'innovation, l'innovation organisationnelle et sociale, la transmissibilité de l'innovation.

\subsection{Aspects théoriques de l’innovation}

De petits changements dans la technologie et dans l'organisation des relations interpersonnelles sur les marchés ou ailleurs peuvent conduire à des innovations « suffisamment bonnes » concernant des coûts d'innovation moindres, tandis que de grands et moyens changements dans la technologie pourraient être qualifiés d'innovation frugale [ZES 14].

La théorie de l'innovation fait souvent la distinction entre les innovations incrémentales et radicales [ETT 11]. Par exemple, les changements dans la forme du guichet automatique ou de son écran visuel sont des innovations incrémentales. L'innovation radicale et l'innovation disruptive sont probablement le concept le plus étudié [KOV 19]. Les innovations radicales se concentrent sur les capacités et les personnes, tandis que les innovations disruptives se concentrent sur les modèles commerciaux [HOP 18]. Au sein des innovations radicales qui impliquent une technologie substantiellement nouvelle, certains auteurs distinguent les innovations disruptives (de rupture) et de maintien en termes de marchés ciblés par les challengers ou les opérateurs historiques [GOV 11]. Poursuivant l'exemple des guichets automatiques, rappelons que les banques qui en disposent offrent une forme d'innovation de maintien vecteur d'avantage concurrentiel, car cela leur permet de répondre n'importe où, n'importe quand, aux clients mobiles contrairement aux concurrents qui n'en mettent pas à disposition [BAT 09].

De nombreuses entreprises mettent l'accent sur la rapidité de l'innovation pour pouvoir tirer parti du démarrage, mais cela comporte des risques et des coûts [ELL 17].

\subsection{L'innovation organisationnelle et sociale}

L'adoption des innovations technologiques émergentes nécessite presque toujours d'innover au niveau organisationnel. Les guichets automatiques permettant la distribution des espèces à moindres coûts n'importe où, n'importe quand [BAT 09] requièrent des changements dans l'organisation des banques. L'avantage concurrentiel obtenu par les banques utilisant les télex pour transférer de l'argent, entraîne à son tour un changement opérationnel majeur qui les oblige à former une alliance coopérative appelée SWIFT [SCO 12]. A l'heure actuelle, toutes ces innovations (ATM, SWIFT) ont été reprises avec succès dans les pays en développement, bien qu'il y ait encore de la place pour la croissance.

L'innovation sociale s'adresse aux besoins sociaux tels que les modèles économiques, les processus démocratiques, la proximité territoriale, l'appropriation collective de la valeur et les considérations environnementales [BIL 19], et plus particulièrement dans le secteur financier à la microfinance [KAP 19] [SUN 15] [VAS 19], au financement participatif [ASS 18], (les obligations à impact social [PAN 18] et à la finance islamique [DAN 20] [MOH 16].

\subsection{La transmissibilité du modèle d'innovation}

Le modèle d'affaires est un concept clé dans les frontières volatiles des sciences de gestion et économiques sur l'innovation. Le modèle d'affaires est censé évoluer avec les changements technologiques, environnementaux et de marché pour formuler de nouvelles propositions de valeur client et de nouveaux étalons de revenus [WIR 18]. Assadi [ASS 20] propose un modèle d'affaires qui regroupe non seulement les trois éléments de valeur client, source de revenus et infrastructure technologique, souvent évoqués dans la littérature spécialisée, mais également le mode de gouvernance pour tenir compte de la réalité de l'entreprise digitale qui se lie à un nombre croissant d'acteurs d'un écosystème en expansion dans le cyberspace. Par exemple, les caisses d'épargne et les banques coopératives, créées au XIXe siècle en Allemagne pour servir les petits épargnants, adoptent des modèles d'affaires différents de ceux des banques commerciales [SCH 16]. Lorsqu'une entreprise 
change d'environnement d'un pays développé à un autre en développement, souvent en créant des filiales [BEN 18], son modèle d'affaires évolue également pour prendre en compte les particularités des capacités cognitives et conatives du nouveau contexte [DAS 17].

Cela vaut également pour les innovations inversées lorsque l'entreprise entreprend de transmettre une innovation d'un pays en développement vers un pays développé [GOV 12] [ZED 15]. Le paiement mobile est un exemple récent, d'abord mis en œuvre aux Philippines, au Kenya, en Afrique du Sud et en Inde [LYM 08], et transmis par la suite dans les pays développés.

\subsection{Les innovations des néo-banques et les stratégies des banques traditionnelles}

Le risque d'innovation étant élevé, l'innovation a souvent lieu dans des start-ups plutôt que dans de grandes entreprises car les créateurs de start-ups sont fortement incités à récolter des bénéfices rapidement et sont donc prêts à prendre des risques élevés [YON 19].

Jusqu'en 2014, 90\% des banques européennes consacrent moins de 0,5\% de leurs dépenses totales à la numérisation et seulement 20 à $40 \%$ de leurs opérations sont numérisées [OLA 14]. Les banques ont des difficultés à innover en interne en raison de l'évolution des réglementations, des processus complexes et de la bureaucratie [CRO 15B]; les technologies héritées qui ne s'intègrent pas aux API [REI 16]; les risques de conformité et de sécurité des données [PWC 19] et la qualité des données disponibles [RYL 20]. Elles utilisent quelques applications mais ne les intègrent pas dans les processus internes et le traitement des données. Elles ne transforment pas non plus les capacités des collaborateurs ou la culture nécessaire pour inaugurer une innovation de processus complète [OLA 14] [PWC 19]. En conséquence, elles proposent une réponse médiocre et lente aux besoins évolutifs de leurs clients [CRO 15A]. Tout cela rend les banques non compétitives, en particulier en raison de la hausse des coûts [RES 18] et des faibles taux d'intérêt sur les prêts [MAR 19].

Le manque d'adaptabilité des banques présente une opportunité pour les acteurs technologiques de se diriger vers des marchés de niche. La presse financière évoque régulièrement les affaires d'un grand nombre de néo-banques dans différents pays. La littérature spécialisée distingue quatre types de néobanques : les courtiers en ligne comme Boursorama et Fortunéo, les affiliées telles que BforBank, filiale du Crédit Agricole et Ma French Bank, filiale de La Banque postale, celles qui proposent des offres numériques dans les réseaux physiques comme Nickel, Orange bank et enfin les banques mobiles comme Revolut et N26 [BEA 18] [CLE 19]. A cette typologie, il convient également de différencier les néo-banques qui fournissent la plupart des services financiers et d'autres qui ne fournissent que les quatre services de base: un compte courant, une carte de débit, un chéquier et la possibilité de déposer ou de payer électroniquement pour les transactions [CLE 19].

Les néo-banques sont des startups agiles. Elles innovent, testent et ne demandent l'approbation qu'ultérieurement [KLI 18] [WIS 15A], alors que les banques traditionnelles doivent attendre préalablement l'approbation réglementaire pour des innovations portant sur les fonctionnalités bancaires sans authentification.

Les néo-banques innovent constamment par l'utilisation des dernières technologies, notamment l'IA et l'analyse de données. Elles utilisent des appareils connectés, adoptent une économie partagée et procèdent à une nouvelle approche pour collecter les données des consommateurs. Ces données sont utilisées pour obtenir des informations et prendre de meilleures décisions, ce qui est apprécié par les consommateurs et les entreprises clientes qui acceptent le partage de données lorsque l'environnement est sécurisé en échange de meilleurs produits et de services mieux adaptés [RES 18].

Cela ne veut pas dire que les banques traditionnelles n'essaient pas d'innover. À la fin des années 1990, WSFS Bank, la plus grande et la plus ancienne société de fiducie gérée localement, crée une entreprise auxiliaire de service et de fourniture d'espèces pour les guichets automatiques non bancaires 
dans toute l'Angleterre, devenue depuis, CashConnect, un générateur des revenus de frais. WSFS Bank fut l'un des premiers investisseurs d'EverBank, lorsque cette banque était encore un concurrent en ligne d'ING. WSFS Bank a récemment déployé une nouvelle fonctionnalité de banque mobile qui permet aux clients de retirer des fonds simplement en agitant leur téléphone sur un écran de guichet automatique. Aucune carte de débit ou code PIN n'est requis [KLI 16].

Les banques traditionnelles copient également certaines innovations [PAR 16] telles que les applications qui permettent aux clients de consulter leurs soldes bancaires et leurs transactions récentes sans entrer de nom d'utilisateur ni de mot de passe [WIS 15A]. Certaines créent leurs propres néobanques ou filiales de banque en ligne afin d'apprendre les subtilités de l'utilisation de la haute technologie, comme TBC qui crée Space en Ukraine [GBN 18].

Les acteurs historiques utilisent surtout des technologies comme l'intelligence artificielle en vue de procéder aux innovations incrémentielles pour des produits et des processus existants desservant des marchés existants, alors que les challengers se concentrent sur les nouveaux produits et marchés donc sur les innovations de rupture [RYL 20].

Les banques peuvent également payer cher pour racheter une néo-banque afin de répondre aux niches et d'accéder à des innovations plus stratégiques. Par exemple, BBVA paie 117 millions de dollars pour acquérir Simple, comprenant une charge de dépréciation du goodwill de 90 millions de dollars [BAR 17]. D'autres achètent la licence d'une technologie à des néo-banques comme Moven ou Seed. Ces technologies peuvent être très simples comme par exemple l'ouverture d'un compte en moins de cinq minutes [WIS 18].

En conséquence, un nombre croissant de prestataires de services financiers trouve qu'il est préférable de collaborer plutôt que de rivaliser avec les acteurs en place [DHA 17]. Cependant des difficultés subsistent dans la collaboration. Premièrement, les grandes banques ont le problème de leurs systèmes hérités, souvent incompatibles avec le système moderne d'une Fintech, situé principalement sur un cloud public. Deuxièmement, les banques traditionnelles mettent du temps pour arrêter leurs relations, souvent de longue date, notamment avec leurs fournisseurs. Troisièmement, les acteurs en place sont plus préoccupés par les questions de réglementation et la gestion des risques et ont du mal à faire des acquisitions, même lorsqu'il s'agit d'innovation qui ne nécessite pas d'approbation réglementaire. Quatrièmement, la preuve de concept n'est souvent pas assez forte pour que les banques traditionnelles s'y penchent rapidement [FIN 418].

L'acquisition directe de la technologie requiert un apprentissage pour l'organisation, mais acquérir une néo-banque nécessite une équipe d'experts en $R \& D$, un apprentissage instantané, ainsi que le pouvoir d'imaginer l'avenir dans un état d'esprit institutionnellement intégré [CRO 18B] [KLI 16] [WIS 15B] et la capture des innovations des challengers acquis. Les banques traditionnelles progressent ainsi rapidement, mais restent préoccupées par l'entrée des entreprises de télécommunications dans le secteur bancaire [PWC 19].

Certaines startups sont également prêtes à être rachetées par les opérateurs historiques en raison des gains immédiats en capital. Toutefois, certains innovateurs sont plus motivés par la passion que par l'argent et cherchent par conséquent à tout faire pour que leur innovation soit un succès. Premièrement, ils élargissent par exemple l'offre de produits [KLI 15] pour accéder à une clientèle plus large [DHA 27] [KLI 15], mais c'est assez difficile pour un challenger dont le budget est limité. Deuxièmement, les challengers tentent d'accéder à une épine dorsale bancaire de base qui est coûteuse et prend du temps à se développer en interne [CRO 15B]. Troisièmement, ils surmontent souvent les barrières réglementaires dans le secteur bancaire [DHA 17].

Les clients que la plupart des néo-banques ciblent sont jeunes, citadins et sous-bancarisés [RES 14] comme par exemple les étudiants [YUR 15]. Le tableau 1 récapitule les défis rencontrés et les solutions apportées par les néo-banques. 


\begin{tabular}{|c|c|c|}
\hline Cible & Problème & Solution \\
\hline Jeunes & Dette, paiements impulsifs & Carte prépayée \\
\hline Jeunes et sous bancarisés & Analphabétisme financier & $\begin{array}{l}\text { Logiciel / API de gestion financière } \\
\text { personnelle }\end{array}$ \\
\hline Jeunes et sous bancarisés & Incapacité d'épargner & Compte d'épargne \\
\hline Jeunes et sous bancarisés & $\begin{array}{l}\text { Incapacité de faire des } \\
\text { transactions }\end{array}$ & Compte de chèque \\
\hline Clients insatisfaits & $\begin{array}{l}\text { Banques lentes, la technologie } \\
\text { est ajoutée et interagit } \\
\text { lentement avec les logiciels } \\
\text { héréditaires }\end{array}$ & Première technologie numérique \\
\hline Voyageurs & Retraits dans différentes devises & Comptes multidevises \\
\hline $\begin{array}{l}\text { Clients de la classe moyenne } \\
\text { ignorés }\end{array}$ & $\begin{array}{l}\text { Ignorances des attentes des } \\
\text { clients; procédures non flexibles }\end{array}$ & $\begin{array}{l}\text { Recherche active des commentaires } \\
\text { de clients }\end{array}$ \\
\hline Clients soucieux des coûts & $\begin{array}{l}\text { Exigences de dépôt minimum, } \\
\text { frais pour comptes d'épargne }\end{array}$ & $\begin{array}{l}\text { Fournir des services de base } \\
\text { gratuitement }\end{array}$ \\
\hline
\end{tabular}

Tableau 1. Exemples de marchés de niche ciblés par les néo-banques. Source: Créé par les auteurs à partir de : [CRO 18A] [KLI 15] [WIS 14]

\subsection{La proposition d'un cadre conceptuel}

Quatre enjeux majeurs ressortent des analyses conceptuelles qui précèdent : les banques traditionnelles ont des difficultés à innover de manière proactive ; un grand nombre de challengers de technologie financière pénètrent les niches non ou mal desservies par les grands ; certains acteurs trouvent la solution dans les collaborations et les alliances ; les banques traditionnelles adoptent différentes stratégies de riposte pour accéder aux nouvelles technologies et aux nouveaux marchés.

Nous postulons que les opérateurs historiques s'engagent généralement dans un processus d'acquisition de l'innovation que nous appelons la « capture d'innovation ».

La figure 1 illustre ce concept. Les institutions bancaires traditionnelles laissent le petit poisson qu'est l'opérateur agile utiliser les nouvelles technologies pour créer des innovations disruptives (de rupture). Elles jettent ensuite leur filet de pêche pour récupérer certaines de ces innovations radicales pour maintenir leur avantage concurrentiel. 


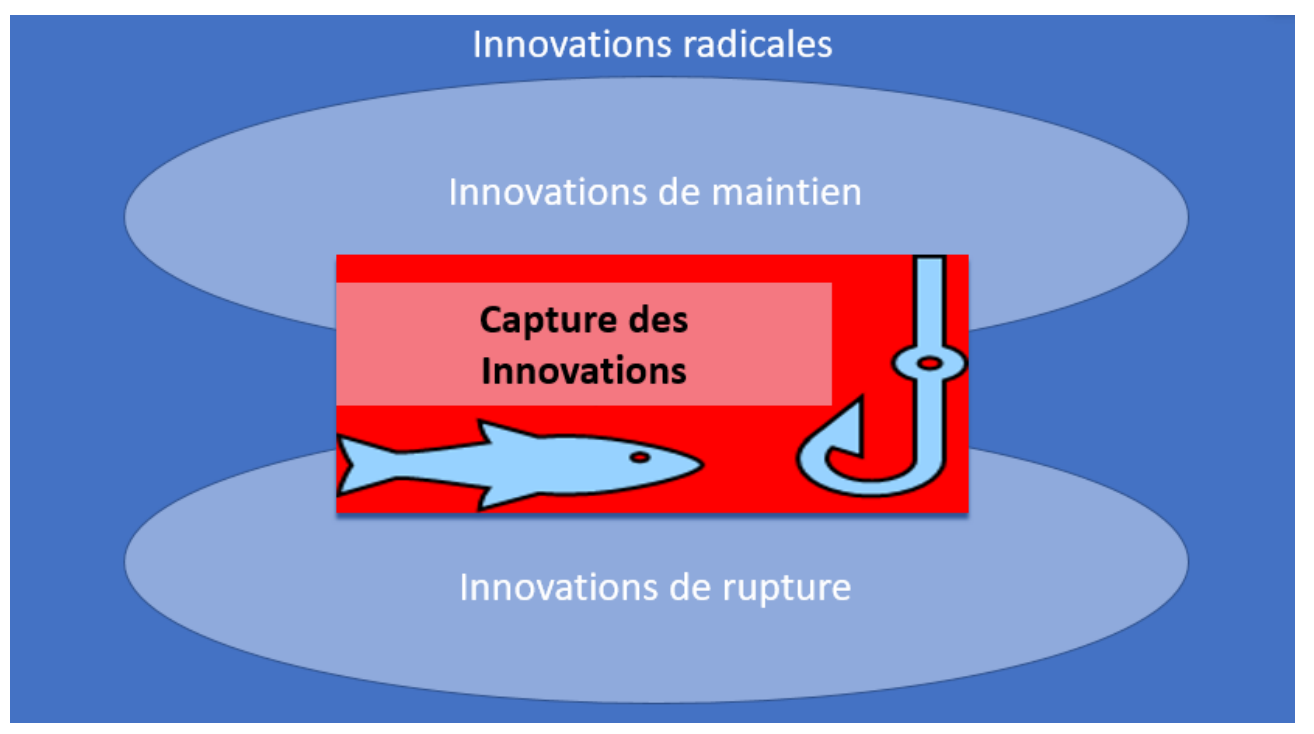

Figure 1. La capture d'innovation : les acteurs historiques repèrent les challengers qui perturbent le marché. Source: conceptualisation des auteurs

Nous appliquons ce cadre conceptuel dans la section suivante à l'étude de cas d'une néo-banque, rachetée par un leader. Il peut être pertinent pour les pays en développement d'encourager de telles initiatives.

\section{L'étude de cas d'une néo-banque française : Nickel}

La méthode de cas exploratoire que nous adoptons ici permet d'obtenir de nouvelles idées en vue de proposer des modèles et éléments théoriques. Nous procédons à l'étude de cas d'une néo-banque, innovation récente de l'offre des services par le biais de cartes et de transactions mobiles. Le cas sélectionné est la première néo-banque en France : Nickel.

La méthode de cas repose notamment sur les sources secondaires pertinentes telles que les revues de presse et des informations sur le site Internet de Nickel (https://nickel.eu/fr), celles de BNP Paribas, les statistiques tirées des rapports annuels ou des médias, le compte de résultat tiré des dépôts légaux et les autobiographies des deux principaux fondateurs [LEB 13].

Les informations ainsi collectées sont complétées par celles de sources primaires, recueillies lors d'un entretien en ligne d'une heure avec Thomas Courtois, président de Nickel Bank.

\section{Les problèmes sociaux :}

Il existe deux problèmes sociaux importants qui sont en amont de cette étude de cas. Premièrement, comme mentionné précédemment, les banques ferment leurs agences dans les zones rurales et les petites villes. L'annexe 1 indique que dans l'ensemble des 28 pays à revenu élevé d'Europe et d'Amérique du Nord pour lesquels des statistiques étaient disponibles, le nombre d'agences bancaires pour 100000 habitants a diminué entre 2008 et 2018, est passé de 49,5 succursales à 31,4 succursales, soit une réduction de $35 \%$. Conformément à cette tendance générale, cette désertification de la clientèle a été plus faible en France, le nombre d'agences pour 100000 clients a baissé de 21,7\% sur la période. Le graphique suivant montre le nombre d'agences en 2016 ainsi que le nombre prévu d'agences bancaires en 2020 de cinq grandes banques de détail françaises, confirmant que les tendances sont également observées au micro-niveau. 


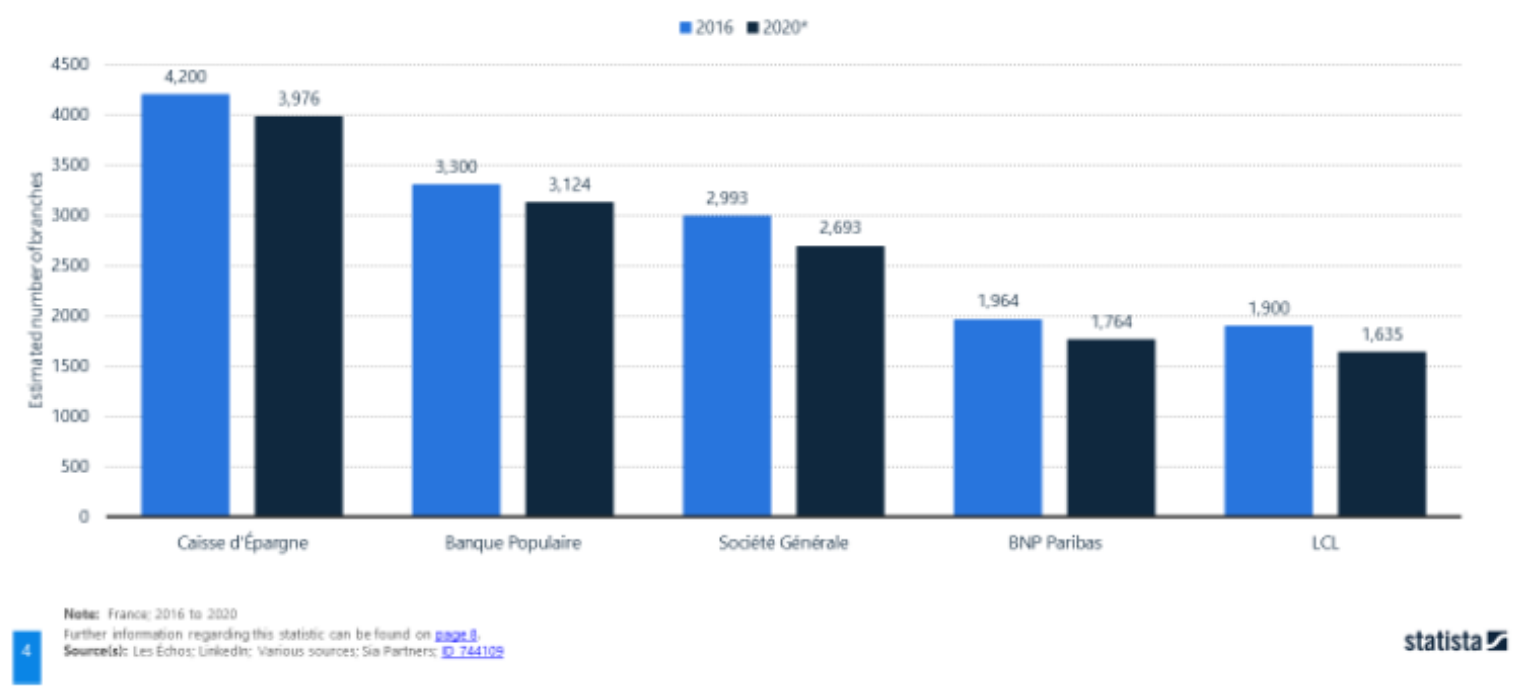

Figure 2. Nombre d'agences en 2016 et 2020 par banque. Source, Statista 2020.

Le deuxième problème social, le surendettement, est un problème important en France, puisqu'il concerne 3\% des ménages: environ 163000 nouveaux cas de surendettement déclarés en 2018 et 762000 ménages sont en processus de désendettement [BAN 18] par rapport à une population française de 29 millions de ménages [INS 19]. C'est une cause de détresse, qui peut être corrélée à des pensées suicidaires chez les femmes [ONS 16]. On considère souvent que l'endettement peut provenir de chocs externes, du cycle de vie (enfants à charge par exemple), de problèmes de maitrise de soi [CAM 90] et d'un biais pour la consommation actuelle [BAR 18] [WEB 01]. Puisque le surendettement conduit souvent à l'interdiction des chéquiers, il diminue la facilité financière des personnes et conduit finalement à l'exclusion financière. Les coûts de transaction pour l'économie peuvent être énormes.

« Nous devons assurer la logistique des transports de fonds, nous devons sécuriser nos locaux, cela demande aussi beaucoup de personnel. De leur côté, les bénéficiaires passent leur vie à attendre... C'est un gros problème pour tout le monde, leur faire un virement serait tellement plus simple » [LEB 13, pp184-85]

Certaines personnes ne sont tout simplement pas en mesure de gérer leurs comptes bancaires et ils émettent des chèques ou utilisent leur carte sans vérifier au préalable d'avoir les fonds suffisants. Ils se voient de ce fait pénaliser de frais bancaires en supplément des intérêts de découvert. Il y a entre 2,5 millions [BAN 19] et 6 millions [LEB 13, p.166] de français considérés comme exclus bancaires. Les décideurs publics, les banques et les clients souhaiteraient donc disposer de produits financiers permettant d'éviter l'endettement. Un moyen de limiter les problèmes de maîtrise de soi est d'interdire d'avoir plusieurs comptes bancaires ou plusieurs cartes de débit ou de crédit [BER 09].

Ainsi, ce que l'on constate, c'est qu'un grand nombre de Français ont des difficultés financières et qu'ils ont de ce fait moins accès aux banques pour tenter de surmonter ces difficultés.

\section{L'idée innovante}

Au début des années 2010, Sogexia, Anytime \& Morning, s'est lancée dans l'industrie du paiement en France [KPM 19]. Ils ont été suivis par Nickel, lancé en 2014. Aujourd'hui, Nickel est la première néo-banque française, la marque utilisée par la Financière des Paiements Electroniques (FPE). La société a été fondée en 2012 par quatre personnes notamment Ryad Boulanouar, ingénieur, et Hugues Le Bret, ancien banquier. L'idée novatrice est synthétisée par Thomas Courtois. 
«Eh bien, l'idée est venue d'un groupe de quatre personnes plus précisément de Ryad Boulanouar, principal fondateur de l'entreprise. Tout d'abord, il a été élevé ici dans une famille modeste, originaire d'Algérie, et a donc vu ses parents gérer leur budget de manière très stricte. Homme d'affaires prospère, il est à l'initiative de la carte cadeau de la FNAC. A partir de cette idée, en se souvenant de comment ses parents géraient leur budget à l'époque, l'idée lui est venue. $\mathrm{OK}$, je vais créer un compte en temps réel sans découvert. Très simple pour les personnes qui ne peuvent pas avoir accès, un accès régulier, à d'autres banques. Et pour les empêcher de surendettement, etc. et pour leur éviter le coût élevé des banques pour les personnes ayant un découvert régulier » (Thomas Courtois, président de Nickel).

Nickel a utilisé une innovation organisationnelle dans l'UE, un établissement de paiement, créé en vertu de la directive européenne sur les services de paiement de 2007. Les établissements de paiement ne sont ni des émetteurs de monnaie électronique (EME) ni des établissements de crédit (banques). Cette forme d'organisation est antérieure aux banques de paiement dans les pays émergents, mais pas aux EME, et aux tests de paiement mobile qui se déroulaient au début des années 2000, ni au modèle de correspondant bancaire qui a débuté au Brésil en 1973 [FAD 09] où les banques ensemble comptaient environ 150000 agents en 2010 [CGA 10].

Nickel a adopté un modèle innovant basé sur un réseau de distribution physique de buralistes (tabacs), où les clients peuvent ouvrir un compte, obtenir une carte de paiement sans découvert autorisé, et un compte bancaire en moins de cinq minutes, sans conditions de revenus. Le buraliste dispose d'un terminal relié à Internet qui permet de vérifier les papiers d'identité, d'ouvrir le compte et d'utiliser la carte de débit. D'une certaine manière, cela ressemble aux correspondants bancaires. Au Brésil, la Caixa Econômica Federal a fait appel aux établissements de loterie comme partenaires. La Banco Brodesco au Brésil a utilisé des bureaux de poste comme agents [IVA 08]. Aux Philippines, un certain nombre de banques s'est associé à l'opérateur mobile SMART et Standard Bank en Afrique du Sud s'est associé à MTN, un opérateur mobile. Au Kenya, Equity Bank s'est associée à la chaîne de magasins de détail Nakumatt. En Afrique du Sud, WIZZIT s'est associé à la chaîne Dunn d'environ 400 magasins de vêtements [IVA 08]. En France, ce sont 10 millions de clients qui passent chaque jour dans les 24500 tabacs français, et attirent une clientèle souvent exclue des banques traditionnelles.

Pour tous ces clients rejetés, Nickel représente une innovation à faible coût. Pour 20 euros par an, Nickel propose un service réduit mais efficace : un compte bancaire, une carte de paiement Mastercard, et la possibilité de déposer des espèces chez les buralistes. Les découverts ne sont pas autorisés, mais cette interdiction évite les frais d'intérêts, qui s'ajoutent aux frais de gestion. Le tableau 2 montre que Nickel ne cible pas une clientèle aisée, mais plutôt une clientèle marginalisée.

\begin{tabular}{|c|c|}
\hline Revenu mensuel & Clients \\
\hline Moins de 500 euros & $22 \%$ \\
\hline Entre 501 et 1000 euros & $30 \%$ \\
\hline Entre 1001 et 1500 euros & $26 \%$ \\
\hline Plus de 1500 euros & $22 \%$ \\
\hline
\end{tabular}

Tableau 1. Profil des clients de néo-banque basé sur les niveaux de revenu. Source: Media reports, 12/2019

Cependant, Nickel a une manière différente de classer ses clients, en indiquant comment ils peuvent percevoir la valeur ajoutée de cette innovation. 
« Nos clients appartiennent à trois catégories. Le premier tiers sont des clients qui ne peuvent avoir accès à aucun autre produit bancaire car ils sont « interdits bancaires » et donc refusés dans toutes les autres banques, mais ils peuvent ouvrir un Compte Nickel. Le deuxième tiers sont des personnes à faibles revenus qui n'ont pas de problème spécifique avec la banque, mais qui veulent gérer leur budget et contrôler le coût de la banque. Le dernier tiers sont des personnes qui choisissent Nickel pour un usage spécifique, pour payer sur Internet par exemple. Ils veulent avoir un compte très sûr pour cela. Parce que le client est débité en temps réel. Instantanément, vous voyez l'opération sur votre compte. Chaque fois que vous payez sur Internet, vous recevez immédiatement une notification sur votre téléphone portable. Voilà donc la première utilisation. Le second est de voyager à l'étranger car nous sommes très compétitifs à l'étranger pour le paiement. » (Thomas Courtois, président de Nickel).

Les deux premières catégories utilisent Nickel comme banque principale. La troisième peut inclure des personnes qui souhaitent contrôler leur besoin de dépenses et des problèmes de maîtrise de soi en ayant des comptes séparés [BAR 18] [BER 09]. Il semble que la plupart des banques purement numériques, telles que Revolut et Monzo, soient généralement utilisées comme comptes secondaires ou tertiaires [ONN 20].

\section{Facteurs clés de succès}

L'idée principale de Nickel est donc venue d'entrepreneurs qui n'avaient pas la banque pour métier, et qui comprenaient les besoins des clients.

« Eh bien, je pense que l'idée est venue de personnes complètement extérieures au secteur bancaire, et je pense que c'est ce qui explique que les quatre fondateurs de Nickel ont pu créer quelque chose de très proche des besoins des clients » (Thomas Courtois, président de Nickel).

L'idée principale est d'avoir une carte où il n'y aurait pas de découvert possible et pas de frais occasionnés par des retards de traitement par la banque, ce qui est fréquent quand les individus ne tiennent pas leurs comptes. Pour Nickel, la transaction est enregistrée en temps réel et le relevé bancaire est mis à jour en temps réel. Cela a donné une nouvelle proposition bancaire à tous ceux qui n'étaient pas satisfaits des banques. Selon Bouchouchi [BOU 17] Nickel «prospère sur un sol fertile: celui de la méfiance des Français envers leur banquier », un sentiment qui fait écho à l'étude rapportée par Nature indiquant que les banquiers ont tendance à tricher beaucoup plus que les autres [COH 14]. De plus, les banques sont lentes car elles effectuent un traitement par lots de données et elles compensent les paiements entre elles, ce qui prend du temps, avant que les transactions n'apparaissent sur le compte d'un client. Le client peut oublier ou faire une erreur de calcul et ainsi dépenser trop. Cela ne peut pas se produire avec Nickel.

« Ce sont des gens qui reprennent le contrôle! L'une des premières raisons invoquées pour ouvrir un compte Nickel est que nous sommes indépendants du système bancaire: nous ne sommes pas une banque! »(Le Bret, cité par [BOU 17]).

Alors que les rapports de presse indiquent que les néo-banques restent deux fois plus chères que les banques en ligne en termes de coûts d'exploitation, Nickel peut avoir des coûts d'exploitation inférieurs, notamment en ce qui concerne les coûts d'acquisition des nouveaux clients.

« Jusqu'à l'année dernière, nous n'investissions pas dans la communication, la publicité, rien. Plus de $60 \%$ de notre client proviennent du bouche à oreilles, et grâce à la puissance de notre réseau de distribution et à notre partenariat avec les buralistes. Ainsi, jusqu'en 2019, notre budget global de communication était inférieur à 1000000 euros, ce qui est très, très différent de toutes les autres néo-banques et banques en ligne. C'est là que Nickel est très, 
très puissant car nous avons un coût d'acquisition très bas et beaucoup, beaucoup plus bas que toutes les autres néo-banques et banques en ligne, car nous n'avons pas eu à investir dans la publicité. Donc, notre coût d'acquisition était d'environ 10 euros contre 30 euros d'après ce que j'ai entendu de Revolut ou N26 et plus de 200 euros pour Boursorama et toutes les banques en ligne, Orange, etc. Le produit était si nouveau, si différent dans le marché que nous avons bénéficié d'une large couverture médiatique sans rien payer! » (Thomas Courtois, président de Nickel).

Dans tous les cas, les néo-banques sont beaucoup plus compétitives que les banques traditionnelles. Leurs trois avantages sont des coûts réduits, une ouverture de compte simplifiée et un compte bancaire de base avec une carte bancaire [KPM 19]. Ils les combinent en offrant des produits de niche de manière innovante, à des marchés de niche. Cependant, la plus grande satisfaction des clients est due à service simple de qualité.

«Le Net Promoter c'est quand vous faites la différence entre les clients qui vous recommandent et ceux qui au contraire vous critiquent. Dans le secteur bancaire, la moyenne se situe entre zéro et moins dix. Pour Nickel, le résultat est de 52. C'est un indicateur de la satisfaction client qui démontre que nos clients sont extrêmement satisfaits. $93 \%$ de nos clients se disent très satisfaits ou satisfaits du nickel. C'est très élevé. » (Thomas Courtois, président de Nickel).

Leurs faibles coûts et leur forte croissance ont permis d'atteindre le seuil de rentabilité en 2018. Le compte de résultat de l'entreprise est présenté dans le tableau 4. Les articles de presse indiquent que l'équilibre est atteint depuis août 2017 [LED 18]. Bien que les pertes cumulées soient de 33 millions d'euros, les promoteurs ont pu trouver des personnes pour investir, avant même que BNP Paribas ne rachète.

\begin{tabular}{|c|c|c|c|c|c|c|c|c|c|}
\hline \multicolumn{10}{|c|}{ Nickel: Some operating and financial results 2015- 2018} \\
\hline & & 2019 & 2018 & 2017 & 2016 & 2015 & 2014 & 2013 & 2012 \\
\hline Employees & Number & 390 & 285 & 199 & 112 & 65 & 33 & 12 & \\
\hline Tobacconists partners * & Number & 5500 & 4335 & 2910 & 2150 & 1120 & & & \\
\hline Clients * & Number & 1500000 & 1135000 & 788000 & 475000 & 200000 & & & \\
\hline Total Deposits in Client Accounts & $€$ Million & & 292.91 & 205.57 & 122.97 & 61.48 & 19.44 & 0.13 & 0.00 \\
\hline Gross Banking Income & $€$ Million & & 59.62 & 39.42 & 21.07 & 9.29 & 2.48 & 0.02 & 0.00 \\
\hline Net Banking Income & $€$ Million & 61.30 & 41.15 & 24.58 & 11.74 & 3.73 & 0.49 & -0.05 & 0.00 \\
\hline Operating Profit & $€$ Million & 6.30 & 1.02 & -2.59 & -6.23 & -6.76 & -7.77 & -5.60 & -3.36 \\
\hline Net Profit & $€$ Million & 4.50 & 1.26 & -2.66 & -6.27 & -8.55 & -7.89 & -5.60 & -3.36 \\
\hline Accumulated Losses & $€$ Million & & -33.07 & -34.33 & -31.67 & -25.40 & -16.85 & -8.96 & -3.36 \\
\hline Share Capital including Share premium & $€$ Million & & 45.03 & 41.25 & 38.94 & 33.86 & 23.53 & 13.02 & 5.61 \\
\hline
\end{tabular}

Tableau 3. Principaux résultats opérationnels et financiers de Nickel

Le succès de Nickel ne vient pas seulement des prix bas, mais aussi de la spécificité du point de vente chez les buralistes : leur plage horaire d'ouverture est bien plus large que celle des banques et cela permet de capter des nouveaux clients qui à la base n'étaient pas entrer dans l'établissement pour cela. En conséquence, ils peuvent guider les clients en cas de besoin et recevoir leurs dépôts. De plus, $45 \%$ des buralistes se trouvent dans des zones rurales où les banques et même les distributeurs automatiques peuvent faire défaut [BRO 13]. Il existe également des synergies pour les buralistes puisque le service supplémentaire leur permet d'attirer des clients qui achèteront autre chose, comme des cigarettes ou des jeux à gratter. Cette stratégie est inédite, les concurrents français et étrangers dépensent beaucoup d'argent pour acquérir de nouveaux clients. Le buraliste gagne 3 euros la première année pour l'ouverture et l'activation du compte et 1 euro les deuxième et troisième années.

«Le premier intérêt est que nous leur apportons de nouveaux revenus car ils sont payés pour chaque opération qu'ils font pour Nickel. Deuxièmement, nous apportons du trafic dans 
la boutique. Nous considérons que lorsqu'un buraliste passe un contrat avec Nickel, le trafic augmente de $7 \%$. Troisièmement, lorsque le client paie avec une carte Nickel dans le magasin de tabac, c'est gratuit pour le buraliste. Alors, ils font des économies. Ils savent que le tabac tue un client. Ils savent que Nickel est bon pour le client et ils en sont très fiers. Oui, très bien. Et ils sont devenus banquiers, vous savez ? Donc, pour certains buralistes, c'est aussi important ». (Thomas Courtois, président de Nickel).

En juillet 2017, la startup a pris un autre avantage stratégique avec l'acquisition de ses fonds propres pour 200 millions d'euros par BNP Paribas (dont 159 millions de goodwill). La valeur de l'entreprise était de 400 millions d'euros [BNP 19]. Ainsi, on peut voir que les startups peuvent générer un énorme profit pour leurs fondateurs, validant la littérature théorique [YON 19]. De plus, cela signifie que la direction clé de Nickel n'a plus besoin de dépenser de l'énergie à la recherche d'investisseurs, tandis que les néo-banques indépendantes telles que Revolut pourraient être en difficulté si elles ne trouvent pas régulièrement de nouveaux investisseurs [ONN 20]. Pour BNP Paribas c'était un moyen de rattraper son retard sur la banque 2.0 car sa propre banque en ligne, Hello Bank, était en difficulté depuis son lancement en 2013. Pour Nickel, cette fusion a permis d'importantes synergies avec sa maison mère dans les achats, la gestion des risques, la conformité, ou la sécurité des données.

«BNP Paribas est très utile pour Nickel. Et surtout, dans trois domaines qui sont la conformité, en particulier la lutte contre le blanchiment d'argent, où ils sont très puissants, et ils nous apportent l'expérience. BNP Paribas est également très utile dans la gestion des risques. Et dans les aspects juridiques. » (Thomas Courtois, président de Nickel).

Les faibles taux d'intérêt en Europe constituent une menace majeure pour les banques rentables, les obligeant ainsi à réduire leurs coûts. Elles ne veulent plus faire de publicité pour leurs produits d'épargne sur lesquels elles ne font aucun gain, alors que c'était leur principal argument de vente. Chaque banque traditionnelle fonctionnait avec sa propre compagnie d'assurance avec des comptes garantis en euros. Mais encore une fois, à une époque où les taux d'intérêt sont bas, rémunérer ces comptes n'a guère de sens. En conséquence, les banques traditionnelles licencient des employés et ferment même des agences dans les petites villes. La désertification des zones rurales et des petites villes par les banques est à l'origine de la croissance de Nickel. Selon un rapport de la Banque de France publié en juin 2019, près de 3000 guichets automatiques (GAB) ont été fermés entre 2015 et 2018. Par conséquent, de nombreux clients ne bénéficient plus d'un service personnalisé et ils voient peu de raisons de rester client de leur banque historique, avec ses frais de service élevés. Les néobanques mobiles conviviales ont effectivement des coûts d'exploitation bien inférieurs et ont pu profiter de ce phénomène.

Une partie des coûts de fonctionnement élevés des banques traditionnelles est due à la lutte contre le blanchiment d'argent et le financement du terrorisme. Lors de l'ouverture d'un compte en ligne, ces banques traditionnelles doivent demander de nombreux documents, ce qui peut être fastidieux. Au moins deux pièces d'identité sont requises, ainsi qu'une preuve de résidence et un relevé bancaire prouvant que vous avez déjà un compte dans une autre institution. Pour simplifier le processus, chez Nickel, il vous suffit d'une pièce d'identité à scanner au terminal de l'un des 5500 buralistes partenaires agréés par l'ACPL. À l'avenir, la biométrie ou les systèmes centraux tels que FranceConnect qui sont liés à l'administration fiscale, pourraient encore simplifier les contrôles d'identité. Au Royaume-Uni et en Allemagne, on peut prendre un selfie et une photo de son document avec son téléphone. La France devrait copier cela pour rester compétitive.

Même en cette période de COVID 19, Nickel poursuit sa croissance en nombre de clients et en nombre de salariés, même si cette dernière s'est effectivement affaiblie. Les dépenses ont diminué, mais les fonds détenus dans les comptes courants ont augmenté. 
«Eh bien, comme tout le monde, je pense. Ce que nous avons observé, c'est que nos clients ont conservé leurs revenus. Dans l'ensemble, on constate qu'au début du mois d'avril, ils ont reçu à peu près le même montant que le début du mois de mars, donc ils n'ont pas perdu leurs revenus. Ils dépensent $25 \%$ de moins avec la carte de crédit. Et, le volume d'un dépôt ou d'un retrait en espèces a été réduit de $50 \%$. Le montant qu'ils conservent sur le compte a augmenté. » (Thomas Courtois, président de Nickel).

\section{Sources de revenus et business models}

L'offre de Nickel s'accompagne d'une application mobile à partir de laquelle le client peut suivre ses opérations en temps réel, ce qui a constitué une innovation. Le tout pour un abonnement annuel de 20 euros. La néo-banque, FPE, est détenue à 95\% par BNP Paribas depuis avril 2017 [BNP 20]. En mars 2020, il comptait 7000 partenaires buralistes. La confédération française des buralistes détient les 5\% restants. Grâce à ce réseau de distribution existant, la banque française est devenue la première néobanque en France, avec 1,5 million d'ouvertures de comptes enregistrées à ce jour, dont 400000 rien qu'en 2019. Cette performance le place devant l'allemand N26 (1 million en France, 5,4 millions dans le monde) et le britannique Revolut (900 000 en France et 10 millions dans le monde).

Les néo-banques ne permettent pas toutes les transactions sans frais. Le tableau 4 fournit des détails sur certains termes et conditions. Par exemple, les paiements peuvent être gratuits dans la zone euro, mais il peut y avoir des frais pour d'autres transactions internationales. Nickel facture également 1 euro pour chaque retrait dans un distributeur automatique de la zone euro. En 2019, la banque a lancé une carte premium, Nickel Chrome, avec une assurance complémentaire et des paiements gratuits à l'étranger, pour 30 euros supplémentaires par an. L'offre a déjà attiré 125000 clients.

\begin{tabular}{|c|c|c|}
\hline & $\begin{array}{l}\text { Compte NICKEL } \\
\text { standard }\end{array}$ & $\begin{array}{l}\text { COMPTE CHROME } \\
\text { PREMIUM }\end{array}$ \\
\hline $\begin{array}{l}\text { Abonnement à des services de banque à distance } \\
\text { (Internet, téléphone fixe, SMS, etc...) }\end{array}$ & $\begin{array}{l}\text { Abonnement à des services de } \\
\text { banque à distance (Internet, } \\
\text { téléphone fixe, SMS, etc...) }\end{array}$ & idem \\
\hline $\begin{array}{l}\text { Abonnement à des produits offrant des alertes sur } \\
\text { la situation du compte par SMS par mois }\end{array}$ & $\begin{array}{l}\text { Gratuit (Au-delà de } 60 \\
\text { SMS par an : } 1 \text { \$ par } \\
\text { tranche de } 10 \text { SMS } \\
\text { supplémentaires) }\end{array}$ & idem \\
\hline Tenue de compte - abonnement par carte de crédit & $20 €$ & $20 €-30 €$ \\
\hline $\begin{array}{l}\text { Versement d'espèces en Point de Vente NICKEL } \\
\text { (dépôt) }\end{array}$ & $2 \%$ du montant déposé & idem \\
\hline $\begin{array}{l}\text { Alimentation d'un compte NICKEL par } \\
\text { carte bancaire }\end{array}$ & $2 \%$ du montant déposé & idem \\
\hline $\begin{array}{l}\text { Retrait d'espèces dans les distributeurs } \\
\text { automatiques de billets en France et en zone } \\
\text { SEPA }\end{array}$ & 1 Euro par retrait & Gratuit \\
\hline Retrait d'espèces en point de vente Nickel (tabac) & $0,50 €$ & idem \\
\hline $\begin{array}{l}\text { Retrait d'espèces dans un guichet automatique à } \\
\text { l'extérieur de la zone SEPA }\end{array}$ & 2 euros & 1 euro \\
\hline $\begin{array}{l}\text { Paiement par carte de biens et de prestations de } \\
\text { services dans les commerces ou à distance hors de } \\
\text { la zone SEPA }\end{array}$ & $1 €$ par transaction de paiement & Gratuit \\
\hline
\end{tabular}

Tableau 4. Conditions d'utilisation des comptes Nickel. Source : Sur la base du site Web de Nickel (consulté le 20 avril 2020) 
Pour lutter contre le blanchiment d'argent, les dépôts en espèces sont limités à 250 euros par jour et 750 euros par mois. L'offre des néo-banques est avant tout conçue pour faciliter les paiements, avec des cartes bancaires contrôlables du bout des doigts en temps réel et une expérience client différente du monde bancaire traditionnel. Le paiement mobile est un plus : Apple Pay ou Google Pay est accessible dans la plupart des néo-banques. Ainsi, alors que les clients ouvrent un compte pour tester le service, ils domicilient ensuite progressivement leurs revenus pour faire des néo-banques leur banque principale.

Les banques ont également des coûts élevés car les gens sont préoccupés par les problèmes de sécurité même si leurs dépôts sont garantis par le Fonds de Garantie et de Résolution des Dépôts (KPMG, 2019).

« Nous avons toujours de petits litiges, mais très limités. Je pense que ce qui nous est reproché, est identique à l'ensemble du secteur bancaire, pas seulement propres aux néobanques. C'est quand les clients ne sont pas d'accord avec une opération spécifique ou ce genre de choses ». (Thomas Courtois, président de Nickel).

\section{Discussion et conclusion}

\section{Les leçons stratégiques de la capture d'innovations}

Ce cas démontre que les innovations de rupture prospèrent lorsqu'il existe de niches mal desservies ou non desservies par les opérateurs en place. Dans notre cas particulier, les banques en place s'étaient soit retirées des marchés ruraux pour des raisons de coûts, soit elles avaient abandonné les personnes surendettées du fait du risque encouru. Les banques traditionnelles ne répondent pas aux besoins des personnes à faible revenu qui recherchent des produits de base. En conséquence, cette innovation lowcost vient satisfaire un marché en besoin. De plus, Nickel a ajouté une touche humaine à travers les buralistes pour lancer le produit. Pour être rentable, Nickel propose un produit basique bon marché mais facture des frais pour les autres services. Ce modèle de subvention croisée est particulièrement pertinent dans les pays pauvres pour permettre l'inclusion financière concernant les services de paiement de base et l'ajout de frais pour le reste de l'offre.

La longue période pour pouvoir ouvrir une banque montre à la fois la prudence et les blocages des institutions conservatrices. C'était le cas par exemple pour avoir l'approbation réglementaire pour cette innovation car les transferts ont été effectués par une agence agréée appelée STET. Cette agence appartient aux banques en place, qui ont refusé de collaborer. Enfin, les challengers ont trouvé une petite banque, le Crédit mutuel Arkéa, qui souhaitait s'engager dans une alliance stratégique. Le challenger doit être motivé et patient pour dépasser la résistance institutionnelle. Les pays en développement peuvent apprendre de cela en facilitant l'accès non seulement dans le secteur de l'agriculture, mais aussi pour les petites entreprises de haute technologie, éventuellement avec des garanties de l'État.

BNP Paribas a déboursé 200 millions d'euros pour récupérer cette innovation. Cela peut provenir de l'utilisation d'un véritable cadre de valorisation des options [DIX 95] [TAN 18]. L'avantage stratégique décrit par ce cadre est que l'investissement dans un projet offre des options d'ajouts ainsi que d'abandon. Il y avait bien sûr aussi des avantages immédiats. Les clients de Nickel (1,5 millions) ont environ 400 millions d'euros sur leur compte bancaire, ce qui est revenu à BNP Parisbas par ce rachat plutôt qu'à un concurrent. L'accès à un tel nombre de clients fournit également des données de transaction qui sont tout aussi précieuses car elles peuvent être utilisées pour l'intelligence artificielle, ainsi que pour réduire les coûts, améliorer les produits et prédire les marchés [RYL 20]. Il existe également des avantages de l'apprentissage organisationnel qui découlent de l'expérience [BAR 95] [HAA 14], que les professionnels de métier ont du mal à se construire. 
«Et sans toute la pollution que les personnes peuvent avoir lorsque qu'elles ont 20 ans d'expérience dans le secteur bancaire, ce qui est mon cas. Donc, je n'aurais probablement jamais inventé le compte nickel. ». (Thomas Courtois, président de Nickel).

Par conséquent, alors que les grandes banques ont des compétences, des connaissances et une perspicacité plus élevées dans leurs domaines [DAS 17] (Daspit, 2017), elles peuvent ne pas être en mesure de visualiser des solutions que des acteurs externes sont capables de trouver. Il est intéressant de la part des opérateurs historiques de laisser grandir les « petits poissons » puis de « capturer » les plus intéressants. C'est ce que nous avons appelé la capture d'innovation.

\section{Problèmes d'innovation sociale}

«L'innovation permet de développer de nouveaux types de parcours clients qui ont un impact positif sur la société. L'offre de services Nickel en France, par exemple, allie innovation et inclusion sociale pour proposer des solutions bancaires accessibles à tous via les buralistes » [BNP 19, p.30].

Il est clair que le rapport annuel de BNP Paribas reflète le discours mondial sur l'inclusion financière et souhaite mettre en évidence cette dimension de responsabilité sociale, même si le total des opérations de Nickel ne représente que 0,1\% des opérations de BNP Paribas [BNP 20].

Le modèle bancaire correspondant au Brésil a montré qu'il est possible de fournir des services financiers à partir de commerçants tels que des épiceries et des pharmacies situées dans des endroits éloignés tant qu'il y a une connexion téléphonique. Depuis, ce modèle a voyagé dans de nombreux pays alors que les prestataires de services financiers examinaient différents réseaux de distribution. L'utilisation des buralistes en France s'inscrit bien dans ce modèle, notamment à la suite de la désertification des petites villes par les banques. Par conséquent, nous voyons une innovation des pays en développement dans les services financiers venir dans un pays développé. Cela devrait s'ajouter à la théorie de l'innovation inversée qui s'est initialement focalisée sur la même entreprise développant un produit dans un pays en développement et le ramenant ensuite dans les pays développés [GOV 12] Ensuite, cette théorie s'est élargie et elle inclut désormais des produits d'autres entreprises dans les pays en développement reproduits dans les pays développés [ASH 21 IN PRESS]. Les idées de services ne peuvent pas toujours être brevetées mais peuvent voyager dans un monde globalisé. Cependant, le créateur de Nickel ne rejoint pas tout à fait nos propos.

« Non. L'idée est née en France et c'est un produit très français. L'idée au tout début était de proposer une solution à des personnes en dehors du système bancaire traditionnel. Oui, donc au tout début le prénom de Nickel était «pas de banque ». Alors, vous voyez ce que c'était. Il a été construit au départ comme une alternative aux banques». (Thomas Courtois, président de Nickel).

Ce qui est néanmoins plausible, c'est que l'innovation et le mimétisme suivent le même chemin. Dans les pays en développement, les pauvres adoptent les paiements mobiles pour les envois de fonds des villes aux villages. Cela a été progressivement imité par les immigrants des pays développés qui envoient désormais de l'argent aux pays en développement en utilisant Internet et de nouvelles applications mobiles, en contournant les banques. Cela a ensuite conduit à l'idée que les banques pourraient être contournées pour les paiements, même dans les pays développés.

«Les travailleurs immigrés des pays développés envoient depuis longtemps par Wester Union ou MoneyGram des espèces à leurs familles en Afrique, en Europe de l'Est ou, de plus en plus...au Pakistan... C'est d'ailleurs en observant le comportement de ces immigrés et de tous ceux qui vivent en prépayé en France que Ryad avait eu l'idée du compte en bar tabac. " [LEB 13] (Le Bret, 2013, p. 162)

Souvent une innovation provient d'un pays pauvre qui a besoin de choses accessibles et durables, et non d'un prix élevé, pour permettre une stratégie de croissance rapide (blitz-scaling strategies), correspondant à l'innovation de Gandhi. [PRA 10]. L'innovation de Gandhi vient disrupter les business 
models classiques, modifier les compétences organisationnelles et en créer de nouvelles. Certaines innovations nécessitent également des réseaux de distribution différents, comme Nickel l'a fait avec l'utilisation des bureaux de tabac. Ainsi, les innovations de Gandhi ne doivent pas nécessairement être inspirées d'un pays en développement. Dans notre cas, l'innovation fut inspirée par les difficultés rencontrées par les familles modestes en France, elle pourrait peut-être s'étendre aux pays en développement.

En Inde, la création de banques de paiement a été autorisée en 2014. La première a été créé en 2017, et il y a désormais quatre banques de paiement. Chacune d'entre elles agit sur son propre réseau de distribution. India Post Payment Bank (IPPB) utilise La Poste comme réseau de distribution, Paytm utilise son propre réseau de clients, Airtel utilise le réseau mobile des clients, et Fino utilise son réseau d'affaires. Cependant, leur pérennité est incertaine [ASH 20B] [DIA 19] depuis qu'ils ont de multiples réglementations à respecter. Par exemple, alors que l'IPPB avec ses 10 millions de clients en Inde n'a pas encore réalisé de bénéfices, Nickel l'est avec pourtant bien moins de clients. C'est sûrement dû au fait que des frais sont prélevés sur toutes les transactions en dehors des opérations basiques. En Inde, l'Etat a restreint les opérations sur lesquelles des frais peuvent être facturés, il est donc plus difficile pour les opérateurs d'être rentables [ASH 20B].

Une innovation peut donc rencontrer des difficultés dans un pays en développement et bien fonctionner dans un pays développé : c'est un apport intéressant pour la théorie de l'innovation. En effet, dans les pays en développement, les décisions politiques concernant les acteurs du marché semblent empêcher les institutions d'être réellement autonomes. Le rôle des Etats des pays en développement est de faciliter le développement des innovations par la création des systèmes d'innovations nationaux [CAS 17] mais il faudrait ensuite permettre aux Fintech de gérer elles-mêmes leurs business models et accepter de ne pas interférer.

En Europe, le gouvernement français ne peut pas trop intervenir sur le marché de Nickel, car cela donnerait un avantage aux concurrents allemands et britanniques.

Nickel a débuté en tant qu'entreprise sociale avec comme objectif d'être performante tout en faisant $\mathrm{du}$ bien, et dans une perspective de long terme. Les entreprises sociales du secteur financier sont souvent rachetées par les institutions historiques [ASH 20A]. Cela fut le cas du Irish Loan Funds qui a duré de 1720 à 1961 [HOL 98], de l'American Savings Trusts qui a tenu de 1816 à 1980 ([WAD 11], et du Spanish Montes de Piedad à Barcelone qui a duré de 1750 à 1907 [CAR 12], chacune d'entre elles a ensuite été racheté par une banque historique. Nickel est un cas similaire d'acquisition d'une néo-banque par une banque traditionnelle, à la différence près que le rachat s'est fait extrêmement rapidement par rapport aux cas précédemment cités. Peut-être que les banques n'ont fait que se retirer des villages et des petites villes pour regrouper et réorganiser leurs forces sous de nouvelles formes d'organisation, imitant le concept anglais de « battre en retraite » de la guerre du XVIIe siècle. 
[ASS 18] ASSADI, D., ASHTA, A., \& Jung, A. (2018). A Tale of Three Musketeers of Alternative Finance: Stagnating Microcredit, Growing P2P Online Lending and Striving for Slow Money. Journal of Innovation Economics \& Management, 26(2), 13-36.

[BAN 18] Banque de France. (2018). Baromètre du surendettement: 4ème trimestre 2018. Retrieved from Paris:

[BAN 19] Banque de France. (2019). Rapport annuel de l'observatoire de l'inclusion bancaire - exercice 2018. Retrieved from Paris:

[BAR 17] BARBA, R. (2017). Valuing a digital bank isn't so Simple. American Banker, 182(24), 1.

[BAR 18] BARBOZA, G. (2018). I Will Pay Tomorrow, or Maybe the Day After. Credit Card Repayment, Present Biased and Procrastination. Economic Notes, 47(2/3), 455-494. doi:10.1111/ecno.12106

[BAR 95] BARRETT, F. J. (1995). Creating Appreciative Learning Cultures. Organizational Dynamics, 24(2), 36-49.

[BAT 09] BATIZ-LAZO, B. (2009). Emergence and evolution of ATM networks in the UK, 1967-2000. Business History, 51(1), 1-27. doi:10.1080/00076790802602164

[BEA 18] BEAUDEMOULIN, N., BIENVENU, P., \& FLICHE, O. (2018). Études sur les modèles d'affaires des banques en ligne et des néobanques Retrieved from

[BER 09] BERTAUT, C. C., HALIASSOS, M., \& REITER, M. (2009). Credit Card Debt Puzzles and Debt Revolvers for Self Control*. Review of Finance, 13(4), 657-692. doi:10.1093/rof/rfn033

[BET 18] BETARAYA, D. M., NASIM, S., \& MUKHOPADHYAY, J. (2018). Subsidiary Innovation in a Developing Economy: Towards a Comprehensive Model and Directions for Future Research. FIIB Business Review, 7(2), 109125 .

[BIL 19] BILLAUDEAU, V., CHRISTOFOL, H., \& MATHIEU, J.-P. (2019). Dynamiques entrepreneuriales au sein de projets collectifs d'innovation sociale. Marché et organisations, 36(3), 153-177. doi:10.3917/maorg.036.0153

[BNP 19] BNP Paribas. (2019). Document de référence et rapport financier annuel 2018 Retrieved from Paris:

[BNP 20] BNP Paribas. (2020). Document d'enregistrement universel et Rapport Financier Annuel 2019. Retrieved from Paris, France:

[BOU 17] BOUCHOUCHI, C. (2017, mercredi 22 février 2017). Compte-Nickel : le hold-up de la "no bank". L'Obs.

[BRO 13] BROCHEN, P. (2013). Nickel passe la banque au tabac. ÉcoFutur(11 novembre 2013), 1.

[CAM 90] CAMERON, S., \& GOLBY, D. (1990). An Economic Analysis Of Personal Debt. Bulletin of Economic Research, 42(3), 241. doi:10.1111/j.1467-8586.1990.tb00673.x

[CAR 12] CARBONELL-ESTELLER, M. (2012). Montes de piedad and savings banks as microfinance institutions on the periphery of the financial system of mid-nineteenth-century Barcelona. Business History, 54(3), 363-380. doi:10.1080/00076791.2011.638486

[CAS 17] CASADELLA, V., \& UZUNIDIS, D. (2017). National innovation systems of the south, innovation and economic development policies: A multidimensional approach. Journal of Innovation Economics and Management, (2), 137-157

[CGA 10] CGAP. (2010). Update on Regulation of Branchless Banking in Brazil. Retrieved from Washington, D.C.:

[CHR 15] CHRISTENSEN, C. M., RAYNOR, M., \& MCDONALD, R. (2015). What Is Disruptive Innovation? Harvard Business Review, 93(12), 44-53.

[CLE 19] CLERC, L., MORAGLIA, A., \& PEYRON, S. (2019). Les néobanques vont-elles bouleverser leur secteur d'activité ? Revue d'Economie Fnancière(135), 165-180.

[COH 14] COHN, A., FEHR, E., \& MARÉCHAL, M. A. (2014). Business culture and dishonesty in the banking industry. Nature, 516(7529), 86-89.

[CRO 15A] CROSMAN, P. (2015a). How Banks Can Avoid Being Relegated to Utilities. American Banker, 180(92), 0.

[CRO 15B] CROSMAN, P. (2015b). To Speed Payments, BBVA Opens Its Tech Black Box to Dwolla. American Banker, 180(54), 1.

[CRO 18A] CROSMAN, P. (2018a). The challenger bank Chime says it has 750,000 millennial customers. American Banker, 183(16), 1.

[CRO 18B] CROSMAN, P. (2018b). Moven to receive funding from SoftBank, plans to buy bank. American Banker, 183(22), 1 . 
[DAN 20] DANIEL, I., \& JONATHAN, G. M. (2020). Islamic finance and social finance, an opportunity for social enterprises. European Journal of Islamic Finance, 1-11.

[DAS 17] DASPIT, J. (2017). D. Andreini and C. Bettinelli: Business model innovation: from systematic literature review to future research directions. Journal of Management \& Governance, 21(3), 785-792. doi:10.1007/s10997-017-9388-2

[DHA 17] DHAR, N., HOLLY, T., RYAN, D., \& GALEAZ, G. (2017). Top financial services issues of 2018, PWC. . Retrieved from [Available at] https://www.pwc.com/us/en/financial-services/research-institute/assets/pwc-fsi-topissues-2018.pdf (Accessed: 16 June, 2020).

[DIA 19] DIAS, D., \& STASCHEN, S. (2019). Nonbank E-Money Issuers vs. Payments Banks: How Do They Compare? Retrieved from Washington, D.C.:

[DIX 95] DIXIT, A. K., \& PINDYCK, R. S. (1995). The Options Approach to Capital Investment. Harvard Business Review, 73(3), 105-115.

[ELL 17] ELLWOOD, P., GRIMSHAW, P., \& PANDZA, K. (2017). Accelerating the Innovation Process: A Systematic Review and Realist Synthesis of the Research Literature. International Journal of Management Reviews, 19(4), 510530. doi:10.1111/ijmr.12108

[ETT 84] ETTLIE, J. E., BRIDGES, W. P., \& O'KEEFE, R. D. (1984). Organization Strategy And Structural Differences For Radical Versus Incremental Innovation. Management Science, 30(6), 682-695.

[FAD 09] FADEL RIOLINO, R. P., \& DIAS, D. (2009). Branchless Banking and Consumer Protection in Brazil Retrieved from Sao Paolo, Brazil and Washington, D.C.:

[FIN 18] Fintech Innovation Lab. (2018). Mind the Gap: Addressing Challenges to FinTech Adoption. Retrieved from Available at] https://www.accenture.com/_acnmedia/pdf-74/accenture-FinTech-challenges-adoption.pdf (Accessed: 16 January, 2020).

[GBN 18 ] GBN. (2018). TBC launches cloud-based banking service. In: Global Banking News.

[GOV 12] GOVINDARAJAN, V. (2012). A Reverse-Innovation Playbook. Harvard Business Review, 90(4), 120-124.

[GOV 11] GOVINDARAJAN, V., KOPALlE, P. K., \& DANNEELS, E. (2011). The Effects of Mainstream and Emerging Customer Orientations on Radical and Disruptive Innovations. Journal of Product Innovation Management(s1), 121-132. doi:10.1111/j.1540-5885.2011.00865.x

[HAA 14] HAAK-SAHEEM, W., \& DARWISH, T. K. (2014). The role of knowledge management in creating a culture of learning. Management Decision, 52(9), 1611-1629. doi:10.1108/MD-08-2013-0427

[HOL 98] HOLLIS A \& SWEETMAN A. (1998). Microcredit in Prefamine Ireland. Explorations in Economic History 35(4):347-380.

[HOP 18] HOPP, C., ANTONS, D., KAMINSKI, J., \& SALGE, T. O. (2018). What 40 Years of Research Reveals About the Difference Between Disruptive and Radical Innovation. Harvard Business Review Digital Articles, 2-5. [INS 19] INSEE. (2019). Tableaux de l'économie française: Édition 2019. Retrieved from Paris:

[IVA 08] IVATURY, G., \& MAS, I. (2008). The Early Experience with Branchless Banking. Retrieved from Washington, D.C.:

[KAP 19] KAPOOR, S. (2019). Entrepreneurship for Economic and Social Empowerment of Women: A Case Study of a Self Help Credit Program in Nithari Village, Noida, India. Australasian Accounting Business \& Finance Journal, 13(2), 123-142. doi:10.14453/aabfj.v13i2.8

[KLI 15] KLINE, A. (2015). 5 Reasons You Can't Ignore The Neobanks. American Banker Magazine, 125(4), 16-21.

[KLI 16] KLINE, A. (2016). A Community Banker's Guide To Surviving The Next 184 Years. American Banker Magazine, 126(3), 16-24.

[KOV 19] KOVACS, A., MARUllO, C., VERHOEVEN, D., \& VAN LOOY, B. (2019). Radical, disruptive, discontinuous and breakthrough innovation: More or the same? Academy of Management Annual Meeting Proceedings, 2019(1), 1545-1550. doi:10.5465/AMBPP.2019.272

[KPM 19] KPMG. (2019). Panorama of the French Neobanks. Retrieved from Paris, France:

[LEB 13] LE BRET, H. (2013). No Bank: L'incroyable histoire d'un entrepreneur de banlieue qui veut révolutionner la banque. Paris: Les Arenes, Editions Points.

[LED 18] LEDERER, E. (2018, 15 novembre 2018). Nickel, les bons comptes du compte sans banque, p. 13. 
[LYM 08] LYMAN, T. R., PICKENS, M., \& PORTEOUS, D. (2008). Regulating Transformational Branchless Banking: Mobile Phones and Other Technology to Increase Access to Finance. Retrieved from Washington, DC:

[MAR 19] MarketLine. (2019). Lloyds Banking Group Plc SWOT Analysis.

[MOH 16] MOHSIN, M. I. A., \& ALHABSHI, S. O. (2016). Hybrid model of zakah, waqf, qard-hassan \& Islamic finance for a just and sustainable microfinance. Sahulat: A Journal of Interest free Microfinance, 4(1), 27-46. doi:http://sahulatjournal.com/wp-content/uploads/_mediavault/2019/01/Hybrid-Model-of-Zakah-Waqf-QardehasanIslamic-Finance-for-a-Just-and-Sustainable-Microfinance.pdf

[ONN 20] O'NEILL, D. (2020). Can Europe's neobanks survive the funding shake out? Euromoney(May 14), https://www.euromoney.com/article/b1llz05vrxdfsv/can-europes-neobanks-survive-the-funding-shake-out.

[OEC 20] OECD. (2020). 2019 OECD survey of national innovation statistics and Eurostat's Community Innovation Survey (CIS-2016). from OECD

[OLA 14] OLANREWAJU, T. (2014). The rise of the digital bank. McKinsey Digital(July 1), https://www.mckinsey.com/business-functions/mckinsey-digital/our-insights/the-rise-of-the-digital-bank accessed on June 24, 2020.

[ONS 16] ONS. (2016). SUICIDE Connaître pour prévenir : dimensions nationales, locales et associatives. Retrieved from Paris:

[PAN 18] PANDEY, S., CORDES, J. J., PANDEY, S. K., \& WINFREY, W. F. (2018). Use of social impact bonds to address social problems: Understanding contractual risks and transaction costs. Nonprofit Management \& Leadership, 28(4), 511-528. doi:10.1002/nml.21307

[PAR 16] PARTINGTON, R., \& KAHN, J. (2016). Neobanks Chasing U.K.'s Biggest Lenders Face Battle for Survival. Bloomberg.com, N.PAG-N.PAG.

[PRA 10] Prahalad, C. K., \& Mashelkar, R. A. (2010). Innovation's Holy Grail. Harvard Business Review, 88(7/8), 132141.

[PWC 19] PwC. (2019). Crossing the lines: How fintech is propelling FS and TMT firms out of their lanes. Global Fintech Report 2019. Retrieved from https://www.pwc.com/gx/en/industries/financial-services/assets/pwc-globalfintech-report-2019.pdf:

[REI 16] REICH, J., \& KARKAL, S. (2016). A Neobank's Prognosis for Neolithic Banks. American Banker, 181(108), 1.

[RES 14] Research Markets. (2014). Reimagining the Banking Experience: Analysis of Four Neobank Models - GoBank, Moven, Serve, and Simple by Mary Monahan. In Press release.

[RES 18] Research Markets. (2018). European NeoBanks Market Report 2018 In: Research And Markets.com.

[RYL 20] RYLL, L. R., BARTON, M. E., ZHANG, B. Z., \& MCWATERS, J. (2020). Transforming Paradigms: A Global AI in Financial Services Survey. Retrieved from http://www3.weforum.org/docs/WEF_AI_in_Financial_Services_Survey.pdf:

[SCH 16] SCHMIDT, R. H., SEIBEL, H. D., \& THOMES, P. (2016). From Microfinance to Inclusive Finance: Why Local Banking Works. Weinheim, Germany: Wiley -VCH Verlag.

[SCO 12] SCOTT, S. V., \& ZACHARIADIS, M. (2012). Origins and development of SWIFT, 1973-2009. Business History, 54(3), 462-482. doi:10.1080/00076791.2011.638502

[SEN 11] SEN, T. K., \& GHANDFOROUSH, P. (2011). Radical and Incremental Innovation Preferences in Information Technology: An Empirical Study in an Emerging Economy. Journal of Technology Management \& Innovation, 6(4), 33-44.

[SUN 15] SUN, S. L., \& IM, J. (2015). Cutting Microfinance Interest Rates: An Opportunity Co-Creation Perspective. Entrepreneurship: Theory \& Practice, 39(1), 101-128. doi:10.1111/etap.12119

[TAN 18] TAN, J. J. (2018). Interfaces for enterprise valuation from a real options lens. Strategic Change, 27(1), 69-80. doi:10.1002/jsc. 2181

[TEM 18] TEMRI, L. (2018). Responsible innovation. In D. Uzunidis (Ed.), Collective Innovation Processes: Principles and Practices (Vol. 4, pp. 159-176). London, UK: Wiley.

[UZU 18] UZUNIDIS, D. (2018). Collective Innovation Processes: Principles and Practices: John Wiley \& Sons. 
[VAS 19] VASSALLO, J. P., PRABHU, J. C., BANERJEE, S., \& VOOLA, R. (2019). The Role of Hybrid Organizations in Scaling Social Innovations in Bottom-of-the-Pyramid Markets: Insights from Microfinance in India. Journal of Product Innovation Management, 36(6), 744-763. doi:10.1111/jpim.12504

[WAD 11] WADHWANI, R.D. (2011). Organisational form and industry emergence: Nonprofit and mutual firms in the development of the US personal finance industry. Business History 53(7):1152-1177.

[WEB 01] WEBLEY, P., \& NYHUS, E. K. (2001). Life-cycle and dispositional routes into problem debt. British Journal of Psychology, 92(3), 423. doi:10.1348/000712601162275

[WIR 18] WIRTZ, B. W., \& DAISER, P. (2018). Business Model Innovation Processes: A Systematic Literature Review. Journal of Business Models, 6(1), 40-58.

[WIS 14] WISNIEWSKI, M. (2014). A Neobank that Aims to Protect Millennials from Themselves. American Banker, 179(187), 00.

[WIS 15A] WISNIEWSKI, M. (2015a, 12/18/2015). Moven Nixes Passwords for Most Interactions. American Banker, 180(348), 1;

[WIS 15B] WISNIEWSKI, M. (2015b). What Banks Are Learning from Their Neobank Partnerships. American Banker, 180(F328), 0.

[WIS 18] WISNIEWSKI, M. (2018). This neobank believes it can solve banks' digital onboarding problem. American Banker, 183(42), 1.

[YON 19] YONGLIANG STANLEY, H. (2019). The Locus of Innovation: A Literature Review. Journal of Accounting, Business \& Management, 26(1), 63-69. doi:10.31966/jabminternational.v26i01.399

[YUR 15] YURCAN, B. (2015). Get 'Em While They're Young: Why BankMobile Sought Student Servicer. American Banker, 180(197), 1.

[ZED 15] ZEDTWITZ, M., CORSI, S., SØBERG, P. V., \& FREGA, R. (2015). A Typology of Reverse Innovation. Journal of Product Innovation Management, 32(1), 12-28. doi:10.1111/jpim.12181

[ZES 14] ZESCHKY, M. B., WINTERHALTER, S., \& GASSMANN, O. (2014). From Cost to Frugal and Reverse Innovation: Mapping the Field and Implications for Global Competitiveness. Research Technology Management, 57(4), 20-27. doi:10.5437/08956308X5704235 